\title{
Learning Based Resolution Enhancement of Iris Images
}

\author{
Junzhou Huang, Li Ma, Tieniu Tan, Yunhong Wang \\ National Laboratory of Pattern Recognition, Institute of Automation \\ Chinese Academy of Sciences, P.O. Box 2728, Beijing, 100080, P.R. China \\ \{jzhuang, lma, tnt, wangyh\}@nlpr.ia.ac.cn
}

\begin{abstract}
Iris recognition is one of the most reliable personal identification methods. The potential requirement of obtaining high accuracy is that users supply iris images with good quality. It is thus necessary for an iris recognition system to operate the possibly blurred iris images due to less cooperation of users and camera with low resolution. This paper proposes a new algorithm for resolution enhancement of iris images captured by the low resolution camera in less cooperative situations. The prior probability relation between the information of different frequency bands of iris features useful for recognition is firstly learned. Then, it is incorporated into resolution enhancement algorithms to recover the lost information for the seriously blurred images. A large number of experiments on the CASIA iris database demonstrate the validity of the proposed approach.
\end{abstract}

Keywords:Iris recognition, Super resolution, Image enhancement, Markov network, Circular symmetric filter

\section{Introduction}

With current stress on security and surveillance, automatic personal identification in less cooperative situations has been an important research topic. Iris recognition has been studied for personal identification because of iris' extraordinary structure (one example is shown in Figure 1) and non-invasive characteristics [1][2][3][4][5]. Compared with other biometrics such as face and fingerprints, iris is probably the most attractive [1][2].

There exist some successful commercial systems for iris recognition so far. However, it should be noted that the iris diameter in an iris image must be at least 100 pixels for recognition [3]. Current systems would normally work only if the iris images were taken with the cooperative users from a short distance. This means that these systems greatly depend on the cooperation of the users to capture iris images with good quality. Hence, a problem naturally arises: how can we keep the iris recognition systems working normally if there are only lower resolution iris images? One effective solution is to improve resolution by means of super-resolution image enhancement.

Many approaches have been proposed for image resolution enhancement [6][7][8][9] [10][11][12]. In general, these methods may be roughly classified into reconstructionbased methods and learning-based methods.Relatively speaking, the latter can obtain better results than the former [13]. Learning-based methods try to recognize local features 


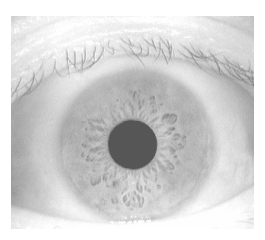

Figure 1: An iris sample

of a low resolution image and then to retrieve the most likely high-frequency information from given samples. It is believed that lost high frequency information can be restored if enough example images are provided. But, common learning-based methods will introduce fake high frequency which has undesirable effects on subsequent iris recognition. So in our modified method, only information useful for recognition is learned and enhanced. Experimental results demonstrate the validity of the proposed approach.

The remainder of this paper is organized as follows. Related work is summarized in Section 2. Section 3 describes the proposed algorithm in details. Experimental results are presented in Section 4, and Section 5 concludes this paper.

\section{Related Work}

\subsection{Iris Recognition}

Much work has been done in iris recognition. Daugman used multi-scale quadrature wavelets to extract texture phase structure information of the iris and Hamming distance for classification [1][2]. Wildes et al. represented the iris texture with a Laplacian pyramid constructed with four different resolution levels and used normalized correlation for verification [3]. Boles et al. calculated a zero-crossing representation of 1D wavelet transform at a few scales of a virtual circle on an iris image to characterize an iris [4]. Our earlier work adopted a bank of circular symmetric filters to extract the local texture features of the iris [5].

However, all such work depends greatly on a restricted condition that high-quality iris image must be captured. If the iris images were captured in a less cooperative situation, their details would inevitably been lost. Especially, this can greatly affect the performance of recognition. Therefore, how to improve the resolution of iris images becomes an important issue in iris recognition.

\subsection{Super-Resolution}

In computer vision, super resolution is generally thought as inferring the missing highresolution image from the low-resolution image [13][14]. The interest has been driven by a variety of applications such as video surveillance, remote sensing and medical imaging. In general, all these methods are classified to reconstruction based methods [6][7][8][9] and learning based methods [10][11][12].

Tsai and Huang [6] first proposed a super resolution method which processed images in frequency domain. Sauer and Allebach [7] modeled super resolution as an interpolation problem with non-uniformly sampled data. Irani and Peleg [8] proposed an 
iterated back-projection (IBP) method to obtain the high-resolution image by simulating the imaging process via warping so that the difference between the observed and simulated low-resolutions was minimized. Stevenson [9] introduced a maximum-a-posteriori (MAP) method for resolution enhancement. All these methods only make use of weak priors for resolution enhancement.

Recently, Freeman [10] incorporated the relationship between middle frequency and high frequency of example images as prior information, and then the Belief propagation algorithm was used to infer a high resolution image from a given low resolution image. Recently, he further introduced a one-pass algorithm for the same problem [11]. Hertzmann [12] proposed an analogy method. Using only a pair of images as training data, he transformed an input image into the resulting image, which has the same relation as the training pair. These learning-based methods obtained significantly better results than existing reconstruction-based algorithms.

However, when these learning-based methods fully learn the intensity prior between all bands of images, some fake high information will be inevitably introduced into the synthesized high resolution image though these information can give us good visual effect. For our iris recognition scenario, they will deteriorate the recognition performance of the overall system. In this paper, we propose a modified learning-based method which can only enhance the high frequency information useful for recognition. We will discuss our approach in detail in the following sections.

\section{Algorithm}

The proposed learning-based resolution enhancement algorithm is primarily inspired by the recent work of Freeman [10][11]. For iris images, Freeman's method can not effectively improve the recognition performance in a less cooperative situation although the resulting images by his algorithm show good visual effect. Different from Freeman's algorithm [11], our algorithm is developed mainly for the purpose of improving the recognition rate rather than enhancing the visual effect. We thus use iris features extracted by a Circular Symmetric Filter [5](instead of intensity of images as used by Freeman)to express the local relation between low frequency information and high frequency information. Such processing can greatly improve the accuracy and efficiency of iris recognition.

In the subsection below, we provide a brief introduction to our method for iris recognition, including image preprocessing and feature representation. More attention is paid how to construct the relation between low and high frequency feature information by training and incorporate this relation into inference of high frequency information.

\subsection{Preprocessing}

As shown in Figure 2a, an iris image contains not only the regions of iris but also eyelid, pupil, etc. In non-cooperative situations, any changes of the users may result in the possible variations of the iris size. Also, the intensity of an iris image is not uniformly distributed because of the illumination variations. For reducing the influence of these factors, the original images should be preprocessed to localize and and normalize the iris.

We can approximately regard the inner and outer boundaries of an iris as circles. Iris localization includes simple filtering, edge detection and Hough transform [5]. Figure 2b 


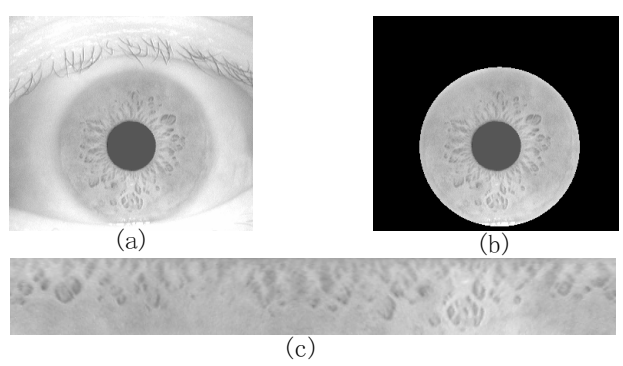

Figure 2: Iris image preprocessing. (a) Original iris image, (b) Localized iris image, (c) Normalized iris image

shows one example of iris localization. We can find that the iris is exactly localized in the image.

The size of the iris in an image is usually affected by many factors such as different subjects and illumination. To achieve accurate recognition results, compensating these changes is necessary. We thus anti-clockwise map the iris ring to a rectangular block of iris texture, with a fixed size. The example of iris normalization is shown in Figure 2c. More details of preprocessing may be found in [5].

\subsection{Filtering}

Because our task is to infer lost information for iris recognition and the recognition results are only affected by iris features, we only concern iris feature information in the whole image. In addition, the majority of useful information of an iris is in a frequency band of 3 octave [2]. A bank of circular symmetric filters can thus be constructed to capture them [5]. The circular symmetric filter (CSF) is developed on the basis of Gabor filters, but there are some differences between them. The former is modulated by a circular symmetric sinusoidal function, while the latter is modulated by an oriented sinusoidal function [5][15]. A CSF is defined as follows:

$$
\begin{gathered}
G(x, y, z)=\frac{1}{2 \pi \sigma_{x} \sigma_{y}} \exp -\frac{1}{2}\left(\frac{x^{2}}{\sigma_{x}^{2}}+\frac{y^{2}}{\sigma_{y}^{2}}\right) M(x, y, f) \\
M(x, y, f)=\cos \left(2 \pi f\left(\sqrt{x^{2}+y^{2}}\right)\right)
\end{gathered}
$$

where $M(x, y, f)$ is the modulated function, $f$ is the frequency of the sinusoidal function. $\sigma_{x}$ and $\sigma_{y}$ are the space constants of the Gaussian envelope along the $x$ and $y$ axis respectively. We can obtain a band-pass filter with a specific center frequency by setting the frequency parameter $f$. The choice of the parameters in (1) is similar to that of [5]. The circular symmetric filter can capture the information of an image in a specific frequency band, whereas it can not provide orientation information because of its circular symmetry. Here, we utilize the CSF to filter iris images for acquiring iris features. 


\subsection{Training}

This step is crucial for the proposed algorithm because the relation between the lowresolution image and the high-resolution image is learned in this step. The training set is composed of the search vector and the high-resolution patch of iris feature. The search vector is composed of the low-resolution patch of iris feature and the overlapped region of the high-resolution patch.

For constructing the training database, we first blur the iris images and scale them down. Then, we can utilize an interpolation method to create a low-pass image of the original image. The interpolated image is degraded in a manner corresponding to the degradation we plan to undo in the process of inferring. The high-frequency band is constituted by the difference between the interpolated version and the original version. Using the CSF, we can obtain iris feature information of the interpolated image as the medium band information. Figure 3 (a) shows a low-resolution iris image, (b) shows the initial cubic interpolated image of low-resolution, (c) shows the high-resolution version of the image, (d) shows the high frequency image and (e) shows the filtered medium frequency image.

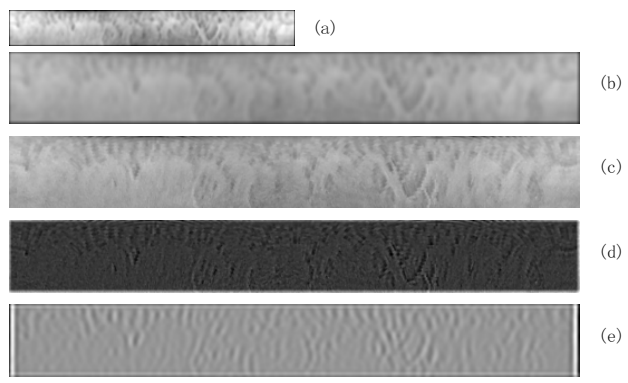

Figure 3: The iris image samples for training

After we have processed the images according to the above steps, we break the built images into patches. Each low-resolution patch is associated with a high- resolution one, centered on the same pixel. In addition, each high-resolution patch overlaps with other neighborhood patches by several pixels. We model the spatial relationships between these patches using a Markov Network [16].

Based on the knowledge of the MRF model, we propose a fundamental assumption that the high frequency information of an iris image only depends on the feature information of interpolated image. This assumption allows the algorithm to be able to work only in two bands. We also assume that the local image contrast does not affect the relation between the two working bands.

Each patch is normalized by a local contrast normalization method, which the highresolution and low-resolution patches are divided by a local energy. Then, we undo the normalization after the matching process to acquire the right high-frequency patch of the input. Normalization can reduce the variability and increase the efficiency of the training set.

The following summarizes the major steps of training data :

1. First, remove the high frequency information and leave an intermediate image for each image in the training set. 
2. Construct an iris feature image by filtering the intermediate image using a CSF. The feature image is thought as the medium frequency information for learning.

3. Use a local contrast normalization method to normalize both the high-resolution and the low-resolution patches.

4. Extract patches from the normalized medium frequency image and the corresponding high frequency image. The patches from the medium frequency image and the corresponding patches from the high frequency image are concentric.

5. Build an index between the vector $X_{k}$ and the vector $Y_{k}$. Here, $X_{k}$ denotes the high frequency patches, $Y_{k}$ denotes the medium frequency information and $k$ denotes the number of the patches in the database.

6. Use the vector $X_{k}$ and the vector $Y_{k}$ to build the search vector. The search vector is composed of the low-resolution patch of the iris feature and the overlapped region of the high-resolution patch.

\subsection{Inference of high frequency information}

This step first obtains the interpolated version of the low-resolution image by cubic interpolation. Then, we can extract the medium frequency information from the interpolated image with a CSF. Broken into patches, the medium frequency image is scanned in a raster-scan order to predict the high-resolution patches at each stage. Finally, the corresponding high-resolution patches predicted from the training set are added to the original test patches. The output is the sum of the high frequency information predicted from the training set and the original test image.

The process of inferring is based on spatial and frequency constraints. The frequency constraint is that the predicted high-resolution patch of iris feature must be linked to a low-resolution patch of iris feature in the training set which should be close to the lowresolution test patch of iris feature. The spatial constraint is that the newly predicted high-resolution patch of iris feature should match with the previously predicted one. A weighting factor is used to control the relative importance of these constraints. The flow chart is shown in Figure 4.

For a typical patch $X$ in the medium frequency information of an input image, we always can find a low-resolution patch in the training set to match it. The match process uses L1 distance because the iris recognition uses this distance to produce the feature vector, which is the average absolute deviation (AAD) of each patch [5]. It is defined as follows:

$$
V=\frac{1}{N}\left(\sum_{N}|f(x, y)-m|\right)
$$

where $N$ is the number of the pixels in the image patch, $\mathrm{m}$ is the mean of this patch, and $f(x, y)$ is the value of iris feature image at point $(x, y)$. The match function to determine the optimal matching patch is given by

$$
M_{K}=\left\|V-V_{K}\right\|
$$




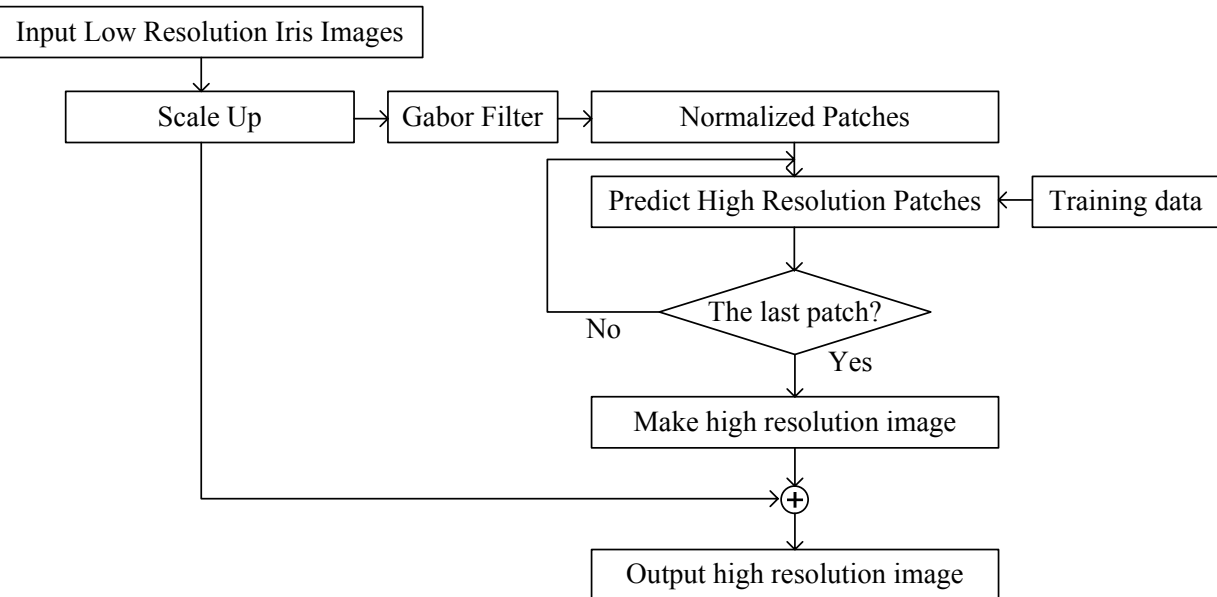

Figure 4: The flow chart of our algorithm

However, only using this function, we can not find the plausible high-resolution patch for each patch of the input image. According to the spatial constraint, to ensure spatial consistency between adjacent patches, we should repeatedly infer patches at their border. A weighting factor $\alpha$ is controlled to adjust the relative importance of two constraints. The whole function is described by:

$$
W_{K}(x)=M_{K}+\alpha H_{K}
$$

$H_{k}$ measures the sum-squared difference of intensity value in the overlapped regions. We can resolve it with an approximation procedure.It is summarized as follows:

1. Scale the test image up by cubic interpolation.

2. Filter the interpolation image of the input by the CSF to make its features.

3. Break the obtained medium frequency image in a raster-scan order, and normalize the low-resolution patches by a local contrast normalization method.

4. Scan over each low-resolution patches. Use (3) to find 200 patch pairs from the training set whose feature vectors are most close to the input patch at each step.

5. Use (4) to select the best matching pair from this sub-set patch pairs.

6. Reverse the normalization for the predicted high-resolution patch and add it to the corresponding output patch. The average pixel values are used in overlapped region.

7. Acquire the desired super-resolution image by adding the high frequency image to the test image. 


\section{Experimental results}

Our experiments are conducted with a large number of iris images in the CASIA data set (unlike fingerprint and face, there is no reasonably sized public-domain iris database). The CASIA iris image database contains 134-class iris images. The total number of iris images is 1088 . They are from 109 different volunteers and captured by a digital optical sensor in two different stages. Since image enhancement is only one of the important parts of an iris recognition method, the proposed method is evaluated by analyzing the recognition performance changes of our previous recognition algorithm [5] with different enhancement methods.

We randomly select 37-class of iris images to form the training data, and other 97class iris images to form the test data. For each iris class in test data, we randomly choose one iris image captured in the first stage as reference image, and other iris images taken in the second stage serve as test samples. Each sample is separately degraded by convolution with three Gaussian kernels and down-sampled to three low-resolution images. The blur degree of the third sequence is relatively maximal. Then, we respectively set these three sequences of degraded images as three groups of test images in order. These three sequence are arranged according to their degradation degree. In this paper, we choose 7 $\mathrm{x} 7$ ( pixels) as the patch size and 2 pixels as the the number of overlapping pixels. Then, the training set contains 45,288 patches.

Figure 5 shows the comparison results of an example in terms of visual effect. Both our result and Freeman's result obtain more impressive visual effects than interpolation result. In whole images, there is not obvious difference between Freeman's result and our result. However, from corresponding patches shown in Figure 5(a), 5(b), 5(c) and $5(d)$, we can see that they are different. The enhancement result of our algorithm is more similar to original high resolution patch than those of Freeman's method.

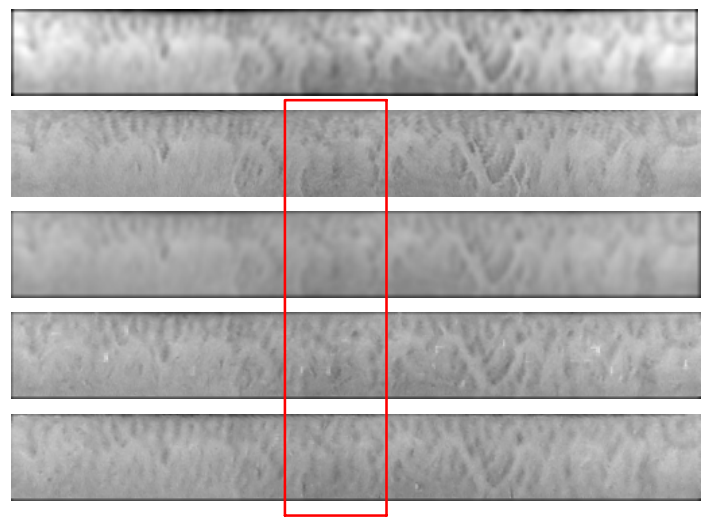

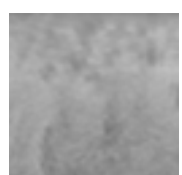

(a)

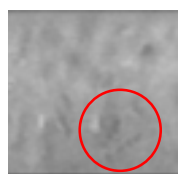

(c)

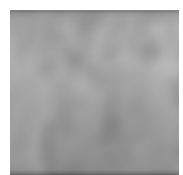

(b)

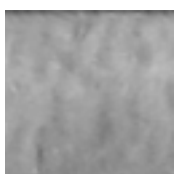

(d)

Figure 5: The comparative results in visual effects. (a) original, (b) interpolation result of low-resolution input, (c) Freeman' result, (d) our result

Table 1 gives the identification results. From it, we can see that our algorithm can obtain the best identification result. Moreover, with the increase of blur degree of the iris image, our algorithm obtains more steady recognition result when the accuracy of the recognition results of both the interpolation method and Freeman's method decrease 
rapidly.

Figure 6 describes the verification results. With the increase of the blur degree, our algorithm always seems to be more advantageous than others. Experimental results show our algorithm can improve the robustness of iris recognition and is much better than Freeman's algorithm in the area of iris image resolution enhancement.

Table 1: Correct classification rates

\begin{tabular}{|l||c|c|c|c|}
\hline Group & Interpolation & Freeman & Our Algorithm & Original \\
\hline \hline Group1 & $73.56 \%$ & $97.70 \%$ & $98.86 \%$ & $100 \%$ \\
\hline Group2 & $44.83 \%$ & $81.61 \%$ & $96.56 \%$ & $100 \%$ \\
\hline Group3 & $35.63 \%$ & $56.32 \%$ & $89.66 \%$ & $100 \%$ \\
\hline
\end{tabular}

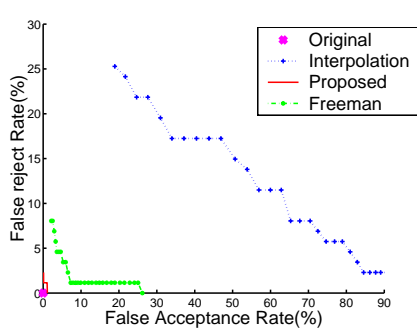

(a) Group 1

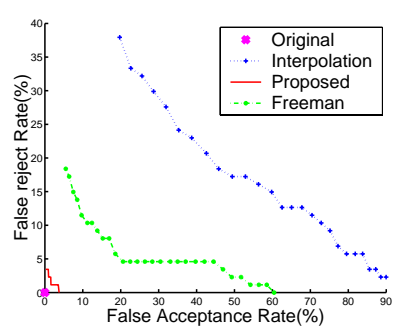

(b) Group 2

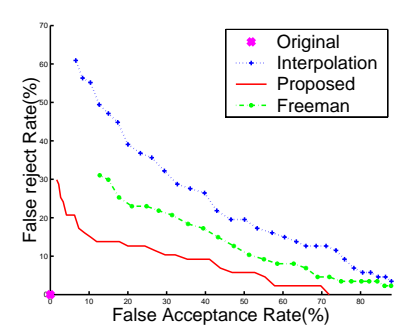

(c) Group 3

Figure 6: Verification results. (FAR vs FRR)

\section{Conclusion}

This paper proposes an algorithm to enhance the iris image so that iris images with poor quality can be accepted by an operational iris recognition system. This algorithm predicts the prior relation between iris feature information of different bands and incorporates this prior into the process of iris image enhancement. Experiment results have shown that this algorithm is successful in both visual effect and recognition rate. Compared with a recent algorithm proposed by Freeman in the area of learning-based super resolution, this algorithm has greatly improved the recognition rate of iris recognition systems. A most important factor that contributes to the good performance of this algorithm is that only those of feature information useful for recognition are processed in learning.

In future work, we should extend our experiments on a larger database. All of our results are based on simply registered iris images [5]. We can attempt to apply different registration methods (such as phase-correlation motion estimation [17]) to improve the experiment results. We also plan to use real iris images with poor quality to test the proposed algorithm.

Acknowledgments The authors would like to thank Dongsheng Wang for self-giving discussion and help. This work is funded by research grants from the Chinese National Hi-Tech Research and Development Program (Grant No.2001AA114180). 


\section{References}

[1] J.G. Daugman. High confidence visual recognition of persons by a test of statistical independence. IEEE Trans. PAMI, 15(11):1148-1161, November 1993.

[2] J.G. Daugman. Statistical richness of visual phase information: Update on recognizing persons by iris patterns. International Journal of Computer Vision, 45(1):25-38, 2001.

[3] R.P. Wildes. Iris recognition: An emerging biometric technology. Proceedings of the IEEE, 85(9):1348-1363, September 1997.

[4] W.W. Boles and B. Boashash. A human identification technique using images of the iris and wavelet transform. IEEE Trans. Signal Processing, 46(4):1185-1188, April 1998.

[5] L. Ma, Y. Wang, and T. Tan. Iris recognition using circular symmetric filters. In ICPR'02, pages II: 414-417, 2002.

[6] R.Y. Tsai and Huang T.S. Multi-frame image resolution and registration. In Advanced in Computer Vision and Image Processing, pages 317-339, 1984.

[7] Sauer K. and Allebach J. Iterative reconstruction of band-limited images from nonuniformly spaced samples. IEEE Trans. Circuits System, CAS-34:1497-1505, 1987.

[8] M. Irani and S. Peleg. Improving resolution by image registration. GMIP, 53:231239, 1991.

[9] S. Borman and R.L. Stevenson. Simultaneous multi-frame map super-resolution video enhancement using spatio-temporal priors. In ICIP'99, pages 27-35, 1999.

[10] W.T. Freeman, E.C. Pasztor, and O.T. Carmichael. Learning low-level vision. In ICCV'99, pages 1182-1189, 1999.

[11] W.T. Freeman, Thouis R.J., and E.C. Pasztor. Example based super-resolution. IEEE Computer Graphics and Application, 22(2):56-65, March-April 2002.

[12] Hertsmann A., Jocobs C.E., Oliver N., Curless B., and Salesin D.H. Image analogies. In ACM SIGGRAPH, pages 327-340, 2001.

[13] S. Baker and T. Kanade. Limits on super-resolution and how to break them. IEEE Trans. PAMI, 24(9):1167-1183, September 2002.

[14] C. Liu, H.Y. Shum, and C.S. Zhang. A two-step approach to hallucinating faces: Global parametric model and local nonparametric model. In CVPR'01, pages I:193198,2001

[15] T.S. Lee. Image representation using $2 \mathrm{~d}$ gabor wavelets. IEEE Trans. PAMI, 18(10):959-971, October 1996.

[16] S. Geman and D. Geman. Stochastic relaxation, gibbs distributions, and the bayesian restoration of images. IEEE Trans. PAMI, 6(6):721-741, November 1984.

[17] Stiller C. and Konrad J. Estimating motion in image sequences. IEEE Trans. Signal Processing, pages 70-91, July 1999. 